\title{
THE APPROXIMATION OF ONE-ONE MEASURABLE TRANSFORMATIONS BY DIFFEOMORPHISMS
}

\author{
BY
}

H. E. WHITE, JR.

1. Introduction. A well-known theorem of Lusin states that any measurable real-valued function $f$ defined on Euclidean $n$-space can be approximated by continuous functions in the sense that there is a sequence $\left\{f_{j}\right\}$ of continuous functions such that

$$
\lim _{j \rightarrow \infty} m\left(\left\{p \mid f(p) \neq f_{j}(p)\right\}\right)=0,
$$

where $m$ denotes Lebesgue measure.

In [9] Whitney proved that the real-valued measurable functions which can be approximated in the above sense by continuously differentiable functions are those which have an approximate total differential at almost every point.

The above material leads naturally to the question of whether analogous statements hold for one-one measurable transformations of $U$ onto $U$, where $U$ is an open subset of Euclidean $n$-space. In [1] Goffman proved that any one-one measurable transformation $T$ of the open unit interval $I^{n}(n \geqq 2)$ onto $I^{n}$ can be approximated by homeomorphisms in the sense that there is a sequence $\left\{\phi_{j}\right\}$ of homeomorphisms of $I^{n}$ onto $I^{n}$ such that

$$
\lim _{j \rightarrow \infty} m\left(\left\{p \mid T(p) \neq \phi_{j}(p) \text { or } T^{-1}(p) \neq \phi_{j}^{-1}(p)\right\}\right)=0 .
$$

This paper is concerned primarily with obtaining a result for transformations of $I^{n}$ onto $I^{n}(n \geqq 2)$ which bears the same relationship to Whitney's result as Goffman's does to Lusin's theorem. That is, it is concerned mainly with the determination of which one-one measurable transformations of $I^{n}$ onto $I^{n}(n \geqq 2)$ can be approximated in the sense of the preceding paragraph by $C^{1}$-diffeomorphisms.

2. Preliminaries. For any positive integer $n$, Euclidean $n$-space will be denoted by $R^{n}$ ( $R^{1}$ will also be denoted by $R$ ). Lebesgue outer measure on the family of all subsets of $R^{n}$ will be denoted by $m^{*}$. Lebesgue measure on the family of Lebesgue measurable subsets of $R^{n}$ will be denoted by $m$. The adjective "measurable" will always be used to mean "Lebesgue measurable."

Let $f$ be a real-valued function defined on the open subset $U$ of $R^{n}$. Let $p$ be in $U$. For any $n$-tuple $\left(k_{1}, \ldots, k_{n}\right)$ of nonnegative integers we write

$$
D_{\left(k_{1}, \ldots, k_{n}\right)} f(p) \text { for } \frac{\partial^{k_{1}+\cdots+k_{n} f}}{\partial x_{1}^{k_{1}} \cdots \partial x_{n^{n}}^{k_{k}}}(p) \text {. }
$$

Received by the editors March 25, 1968. 
If $k_{i}=0$ for $i \neq j$ and $k_{j}=s$, then we write $D_{j}^{s} f(p)$ for $D_{\left(k_{1}, \ldots, k_{n}\right)} f(p)$. If, in addition, $s=1$, then we write $D_{j} f(p)$ for $D_{f}^{s} f(p)$. And, if $n=1$, then we write $f^{(s)}(p)$ for $D_{1}^{s} f(p)$.

Let $E$ be a measurable subset of $R$. A point $x$ in $R$ is called a point of density of $E$ if $\lim _{k \rightarrow \infty}\left(m\left(J_{k} \cap E\right) / m\left(J_{k}\right)\right)=1$ for every sequence $\left\{J_{k}\right\}$ of closed intervals such that $x \in \bigcap_{k=1}^{\infty} J_{k}$ and $\lim _{k \rightarrow \infty} m\left(J_{k}\right)=0$.

A function $f$ defined on an open subset $U$ of $R$ is said to have an approximate derivative at the point $x_{0}$ in $U$ if there is a measurable subset $E$ of $U$ such that $x_{0}$ is a point of density of $E$ and

exists.

$$
\lim _{x \rightarrow x_{0}: x \in E} \frac{f(x)-f\left(x_{0}\right)}{x-x_{0}}
$$

From now on, unless otherwise specified, $U$ will denote an open subset of $R^{n}$.

A real-valued function $f$ defined on $U$ is said to have an approximate partial derivative at $p_{0}=\left(x_{1}^{0}, \ldots, x_{n}^{0}\right)$ in $U$ in the direction of the $x_{j}$-axis $(1 \leqq j \leqq n)$ if the function $f\left(x_{1}^{0}, \ldots, x_{j-1}^{0}, \cdot, x_{j+1}^{0}, \ldots, x_{n}^{0}\right)$ has an approximate derivative at $x_{j}^{0}$. The approximate partial derivative of $f$ at $p_{0}$ in the direction of the $x_{j}$-axis is denoted by $D_{j}^{\mathrm{ap}} f\left(p_{0}\right)$. If $j=1$, we write $f_{\mathrm{ap}}^{\prime}\left(p_{0}\right)$ for $D_{1}^{\mathrm{ap}}\left(p_{0}\right)$.

Let $T$ be a transformation of $U$ into $R^{n}$. We write $T=\left(f_{1}, \ldots, f_{n}\right)$ where the $f_{j}$ are the real valued functions defined on $U$ such that $T(p)=\left(f_{1}(p), \ldots, f_{n}(p)\right)$ for all $p$ in $U$. If $p \in U$ and $D_{j} f_{k}(p)$ exists for $j, k=1, \ldots, n$, then the Jacobian of $T$ at $p$ is denoted by $J(T, p)$.

Definition 1. If $p \in U$ and $D_{j}^{\mathrm{ap}} f_{k}(p)$ exists for $j, k=1, \ldots, n$, then the determinant of the matrix $\left[D_{j}^{\mathrm{ap}} f_{k}(p)\right]$ is called the approximate Jacobian of $T$ at $p$ and is denoted by $J_{\mathrm{ap}}(T, p)$.

Now suppose that $T$ is one-one and that $T[U]=V$ is open in $R^{n}$. Following [1], we will say that $T$ is measurable if $T^{-1}[B]$ is measurable for every Borel subset $B$ of $V$ and $T\left[B^{\prime}\right]$ is measurable for every Borel subset $B^{\prime}$ of $U$. As usual, the transformation $T$ is said to have property $(N)$ if $m(T[Z])=0$ for every subset $Z$ of $U$ of Lebesgue measure zero.

In this paper $r$ will always denote either a positive integer or $\infty$, unless explicitly stated otherwise.

Now we have a definition fundamental to the paper.

DEFINITION 2. Let $T$ be an one-one measurable transformation of $U$ onto $U$. Then $T$ is said to be approximately smooth of order $r$ if, for every $\varepsilon>0$, there is a $C^{r}$-diffeomorphism $\phi_{\varepsilon}$ of $U$ onto $U$ such that $m\left(\left\{p \mid T(p) \neq \phi_{\varepsilon}(p)\right\}\right)<\varepsilon$. If $T$ is approximately smooth of order 1 , then we say simply that $T$ is approximately smooth.

Let $E$ be a measurable subset of $R^{n}$. A point $p_{0}=\left(x_{1}^{0}, \ldots, x_{n}^{0}\right)$ in $R^{n}$ is called a point of linear density of $E$ in the direction of the $x_{j}$-axis $(1 \leqq j \leqq n)$ if the set $E\left(p_{0}, j\right)$, defined by $E\left(p_{0}, j\right)=\left\{x \mid\left(x_{1}^{0}, \ldots, x_{j-1}^{0}, x, x_{j+1}^{0}, \ldots, x_{n}^{0}\right) \in E\right\}$, is measurable and $x_{j}^{0}$ is a point of density of $E\left(p_{0}, j\right)$. We will denote by $E^{*}$ the set of all $p$ in $E$ such that $p$ is a point of linear density of $E$ in the direction of the $x_{j}$-axis for 
$j=1, \ldots, n$. Then $m\left(E-E^{*}\right)=0$ (see [7, p. 298]). We will need the following elementary proposition.

Proposition 1. Let $f$ and $g$ be real valued functions defined on $U$. Let $E$ be $a$ measurable subset of $\{p \mid f(p)=g(p)\}$. Suppose that $p_{0} \in E^{*}$ and that $1 \leqq j \leqq n$.

(1) If $D_{j} f\left(p_{0}\right)$ exists, then $D_{j}^{\mathrm{ap}} g\left(p_{0}\right)$ exists and $D_{j}^{\mathrm{ap}} g\left(p_{0}\right)=D_{j} f\left(p_{0}\right)$.

(2) If $D_{j} f\left(p_{0}\right)$ and $D_{j} g\left(p_{0}\right)$ exist, then $D_{j} f\left(p_{0}\right)=D_{j} g\left(p_{0}\right)$.

Definition 3. For an open subset $U$ of $R^{n}$, let $C_{r}(U)$ denote the set of all measurable functions $f: U \rightarrow R$ with the property that, for every $\varepsilon>0$, there is a function $f_{\varepsilon}: U \rightarrow R$ of class $C^{r}$ such that $m\left(\left\{p \mid f(p) \neq f_{\varepsilon}(p)\right\}\right)<\varepsilon$.

THEOREM 1. Let $T=\left(f_{1}, \ldots, f_{n}\right)$ be a one-one measurable transformation of $U$ onto $U$. Suppose that $T$ is approximately smooth of order $r$. Then the following statements hold.

(1) For $j=1, \ldots, n, f_{j} \in C_{r}(U)$.

(2) For $j, k=1, \ldots, n, D_{j}^{\mathrm{ap}} f_{k}(p)$ exists for almost all $p$ in $U$.

(3) $T^{-1}$ has property $(N)$.

(4) If $U$ is connected, then either $J_{\mathrm{ap}}(T, p)>0$ for almost all $p$ in $U$ or $J_{\mathrm{ap}}(T, p)<0$ for almost all $p$ in $U$.

(5) If $m(U)<\infty$, then $T^{-1}$ is approximately smooth of order $r$ if and only if $T$ has property $(N)$.

(6) If (a) $m(U)<\infty$, (b) $T$ and $T^{-1}$ are approximately smooth of order $r$, and $\left\{\psi_{i}\right\}$ is a sequence of $C^{r}$-diffeomorphisms of $U$ onto $U$ such that

$$
\lim _{i \rightarrow \infty} m\left(\left\{p \mid T(p) \neq \psi_{i}(p)\right\}\right)=0,
$$

then $\lim _{i \rightarrow \infty} m\left(\left\{p \mid T^{-1}(p) \neq \psi_{i}^{-1}(p)\right\}\right)=0$.

Proof. Since $T$ is approximately smooth of order $r$, there is a sequence

$$
\left\{\phi_{i}=\left(f_{1}^{i}, \ldots, f_{n}^{i}\right)\right\}
$$

of $C^{r}$-diffeomorphisms of $U$ onto $U$ such that $\lim _{i \rightarrow \infty} m\left(\left\{p \mid T(p) \neq \phi_{i}(p)\right\}\right)=0$. For each $i$, let $A_{i}=\left\{p \mid T(p)=\phi_{i}(p)\right\}$.

Proof of (1). Suppose $1 \leqq j \leqq n$. Then $A_{i} \subset\left\{p \mid f_{j}(p)=f_{j}^{i}(p)\right\}$, so $f_{j} \in C_{r}(U)$.

Proof of (2). Suppose $1 \leqq j, k \leqq n$. By Proposition 1(1), if $p \in A_{i}^{*}$, then $D_{j}^{\mathrm{ap}} f_{k}(p)$ exists and equals $D_{j} f_{k}^{i}(p)$. Since $m\left(A_{i}-A_{i}^{*}\right)=0$ for $i=1,2, \ldots, D_{j}^{\text {ap }} f_{k}(p)$ exists for almost all $p$ in $U$.

Proof of (3). First recall that if $S$ is an one-one measurable transformation of $U$ onto $U$, then $S^{-1}$ has property $(N)$ if and only if, for every measurable subset $E$ of $U$ with $m(E)>0$, we have $m^{*}(S[E])>0$.

So let $E$ be a subset of $U$ of positive measure. Let $i_{0}$ be such that $m\left(E \cap A_{i_{0}}\right)>0$. Since the diffeomorphism $\phi_{i_{0}}^{-1}$ has property $(N)$ (see [6, p. 339]), $m\left(\phi_{i_{0}}\left[E \cap A_{i_{0}}\right]\right)>0$. But then $m^{*}(T[E]) \geqq m\left(\phi_{i_{0}}\left[E \cap A_{i_{0}}\right]\right)>0$. Thus $T^{-1}$ has property $(N)$. 
Proof of (4) Suppose that $U$ is connected. By the proof of (2), $J_{\mathrm{ap}}(T, p)$ is defined for all $p$ in $\bigcup_{i=1}^{\infty} A_{i}^{*}$ and $J_{\mathrm{ap}}(T, p)=J\left(\phi_{i}, p\right)$ for all $p$ in $A_{i}^{*}$. Since $\phi_{i}$ is a diffeomorphism and $U$ is connected, either $J\left(\phi_{i}, p\right)>0$ for all $p$ in $U$ or $J\left(\phi_{i}, p\right)<0$ for all $p$ in $U$. Since $\lim _{i \rightarrow \infty} m\left(U-A_{i}^{*}\right)=0$, either $J_{\text {ap }}(T, p)>0$ for almost all $p$ in $U$ or $J_{\mathrm{ap}}(T, p)<0$ for almost all $p$ in $U$.

Proof of (5). Suppose that $m(U)<\infty$ and that $T$ has property $(N)$. Then $T[E]$ is measurable for every measurable subset $E$ of $U$. Now let $\varepsilon>0$. For any measurable subset $E$ of $U$ let $\mu(E)=m(T[E])$. Then $\mu$ is a finite measure on the family of measurable subsets of $U$. And $\mu$ is absolutely continuous with respect to $m$ since $T$ has property $(N)$. So there is $\delta>0$ such that $m(E)<\delta$ implies that $m(T[E])=\mu(E)<\varepsilon$ (see [7, p. 31]). Now choose $i_{0}$ such that $m\left(A_{i}\right)>m(U)-\delta$ for $i \geqq i_{0}$. Then $m\left(T\left[A_{i}\right]\right)$ $>m(U)-\varepsilon$ for all $i \geqq i_{0}$. Thus, since $T\left[A_{i}\right]=\left\{p \mid T^{-1}(p)=\phi_{i}^{-1}(p)\right\}$,

$$
\lim _{i \rightarrow \infty} m\left(\left\{p \mid T^{-1}(p)=\phi_{i}^{-1}(p)\right\}\right)=m(U) .
$$

Therefore $T^{-1}$ is approximately smooth of order $r$. The converse follows from (3).

Proof of (6). If $\psi_{i}$ is substituted for $\phi_{i}$ in the proof of (5), then we have a proof of (6).

For $p$ and $q$ in $R^{n}$, we denote the Euclidean distance between them by $\|p-q\|$. For $p$ in $R^{n}$ and $e>0$ we let $B(p, e)$ denote the set $\left\{q \in R^{n} \mid\|p-q\| \leqq \varepsilon\right\}$. For $n \geqq 1$, we let $I^{n}$ denote the set $\left\{\left(x_{1}, \ldots, x_{n}\right) \in R^{n} \mid 0<x_{j}<1\right.$ for $\left.j=1, \ldots, n\right\}$ (we also denote $I^{1}$ by $I$ ). For any subset $A$ of $R^{n}$ we denote the closure, interior, and boundary of $A$ by cl $A$, int $A$, and bdry $A$, respectively.

Definition 4. An open subset $U$ of $R^{n}$ is said to satisfy condition (*) if

(1) $U$ is connected,

(2) $m(U)<\infty$, and

(3) there is a $C^{\infty}$-diffeomorphism $\theta$ of $U$ onto $U$ such that $J(\theta, p)<0$ for all $p$ in $U$.

REMARK. The set $I^{n}$ satisfies condition (*) and the only subsets of $R$ which satisfy condition $(*)$ are bounded open intervals.

3. The main theorem.

THEOREM 2. Let $U$ be an open subset of $R^{n}(n \geqq 2)$ satisfying condition (*). Let $T=\left(f_{1}, \ldots, f_{n}\right)$ be a one-one measurable transformation of $U$ onto $U$. Suppose that

(1) $f_{j} \in C_{r}(U)$ for $j=1, \ldots, n$,

(2) either $J_{\mathrm{ap}}(T, p) \geqq 0$ for almost all $p$ in $U$ or $J_{\mathrm{ap}}(T, p) \leqq 0$ for almost all $p$ in $U$, and

(3) $T^{-1}$ has property $(N)$.

Then $T$ is approximately smooth of order $r$.

We prove Theorem 2 via the following lemmas.

LEMMA 1. Let $U$ be an open subset of $R^{n}$ and let $T$ be a $C^{r}$-transformation of $U$ into $R^{n}$. Let $K$ be a compact subset of $U$ such that $T \mid K$ is a homeomorphism and 
$J(T, p) \neq 0$ for all $p$ in $K$. Then there is an open set $V$ such that $K \subset V \subset U$ and $T \mid V$ is a $C^{r}$-diffeomorphism.

This is a special case of [4, Theorem 1.7].

Definition 5. Let $U$ and $V$ be open subsets of $R^{n}$. Let $D_{r}(U, V)$ denote the set of all $C^{r}$-diffeomorphisms $\phi$ of $U$ onto $V$ such that $J(\phi, p)>0$ for all $p$ in $U$. And we denote $D_{r}(U, U)$ by $D_{r}(U)$.

LEMma 2 (MORSE AND HeUBSCH). Let $U$ be an open subset of $R^{n}$ containing the origin and let $\phi$ be a $C^{r}$-diffeomorphism of $U$ onto $R^{n}$ such that $\phi(0)=0$ and $J(\phi, 0)>0$. Let $e>0$. Then there is a positive number $f<e$ and $\phi^{*}$ in $D_{r}\left(R^{n}\right)$ such that

$$
\begin{aligned}
\phi^{*}(p) & =\phi(p) & & \text { if } p \in B(0, f), \\
& =p & & \text { if } p \in R^{n}-B(0, e) .
\end{aligned}
$$

See [3, pp. 21-24] for a proof of Lemma 2. An easy consequence of Lemma 2 is the following.

Lemma 3. Let $U$ be an open subset of $R^{n}$ containing the point $p_{0}$ and let $\phi$ be a $C^{r}$-diffeomorphism of $U$ onto $R^{n}$ such that $J\left(\phi, p_{0}\right)>0$. Let $e>0$. Then there is a positive number $f<e$ and $\phi^{*}$ in $D_{r}\left(R^{n}\right)$ such that $\phi^{*}(p)=\phi(p)$ for all $p$ in $B\left(p_{0}, f\right)$.

LEMMA 4 (MORSE AND HeUBSCH). Let $U$ and $V$ be connected open subsets of $R^{n}$ such that $B\left(p_{0}, e\right) \subset U \cap V$. If $\phi \in D_{r}(U, V)$, then there is $\phi^{*}$ in $D_{r}(U, V)$ and a compact set $K$ contained in $U$ such that $B\left(p_{0}, e\right) \subset$ int $K$ and

$$
\begin{aligned}
\phi^{*}(p) & =p & & \text { if } p \in B\left(p_{0}, e\right), \\
& =\phi(p) & & \text { if } p \in U-K .
\end{aligned}
$$

See [3, pp. 45-47] for the proof of a more general statement than Lemma 4.

LEMMA 5. Let $U$ be a connected open subset of $R^{n}$ and let $\phi$ in $D_{r}\left(R^{n}\right)$ and $B\left(p_{0}, e\right)$ be such that

$$
B\left(p_{0}, e\right) \cup \phi\left[B\left(p_{0}, e\right)\right] \subset U .
$$

Then there is $\phi^{*}$ in $D_{r}\left(R^{n}\right)$ and a compact set $K$ contained in $U$ such that $B\left(p_{0}, e\right)$ $\subset$ int $K$ and

$$
\begin{aligned}
\dot{\phi}^{*}(p) & =\phi(p) & & \text { if } p \in B\left(p_{0}, e\right), \\
& =p & & \text { if } p \in R^{n}-K .
\end{aligned}
$$

Proof. Since $B\left(p_{0}, e\right) \subset U \cap \phi^{-1}[U]$, by Lemma 4, applied to $U, \phi^{-1}[U]$, and $\phi^{-1}$, there is a compact set $K$ contained in $U$ and $\phi_{*}$ in $D_{r}\left(U, \phi^{-1}[U]\right)$ such that $B\left(p_{0}, e\right) \subset$ int $K$ and

Define $\phi^{*}$ by

$$
\begin{aligned}
\phi_{*}(p) & =p & & \text { if } p \in B\left(p_{0}, e\right), \\
& =\phi^{-1}(p) & & \text { if } p \in U-K .
\end{aligned}
$$

$$
\begin{aligned}
\phi^{*}(p) & =\phi\left(\phi_{*}(p)\right) & & \text { if } p \in U, \\
& =p & & \text { if } p \in R^{n}-U .
\end{aligned}
$$

Then $\phi^{*}$ is the required diffeomorphism. 
LEMMA 6. Let $U$ be a connected open subset of $R^{n}(n \geqq 2)$. Let $\left\{\phi_{j} \mid j=1, \ldots, k\right\}$ be contained in $D_{r}\left(R^{n}\right)$ and let $\bigcup_{j=1}^{k} B\left(p_{j}, e_{j}\right)$ be contained in $U$. Suppose that

(1) $\phi_{j}\left[B\left(p_{j}, e_{j}\right)\right] \subset U$ for $j=1, \ldots, k$, and

(2) $\left\{B\left(p_{j}, e_{j}\right) \mid j=1, \ldots, k\right\} \cup\left\{\phi_{j}\left[B\left(p_{j}, e_{j}\right)\right] \mid j=1, \ldots, k\right\}$ is a disjoint family.

Then there is $\phi$ in $D_{r}\left(R^{n}\right)$ and a compact set $K$ contained in $U$ such that

(a) $\bigcup_{j=1}^{k} B\left(p_{j}, e_{j}\right) \subset$ int $K$,

(b) $\phi\left|B\left(p_{j}, e_{j}\right)=\phi_{j}\right| B\left(p_{j}, e_{j}\right)$ for $j=1, \ldots, k$, and

(c) $\phi(p)=p$ if $p \in R^{n}-K$.

REMARK. If $n=1$, then Lemma 6 is false if $k \geqq 2$. This is what prevents the proof of Theorem 2 from holding when $n=1$.

Proof. For $j=1, \ldots, k$, let $B_{j}=B\left(p_{j}, e_{j}\right)$. Since $U$ is connected, $n \geqq 2$, and (2) holds, there are connected open sets $U_{1}, \ldots, U_{k}$ contained in $U$ such that

$$
\left\{U_{j} \mid j=1, \ldots, k\right\}
$$

is a disjoint family and, for $j=1, \ldots, k, B_{j} \cup \phi_{j}\left[B_{j}\right] \subset U_{j}$. For $j=1, \ldots, k$, by Lemma 5 , there is a compact set $K_{j}$ contained in $U_{j}$ and $\phi_{j}^{*}$ in $D_{r}\left(R^{n}\right)$ such that $B_{j} \subset$ int $K_{j}$ and

Define $\phi$ by

$$
\begin{aligned}
\phi_{j}^{*}(p) & =\phi_{j}(p) & & \text { if } p \in B_{j}, \\
& =p & & \text { if } p \in R^{n}-K_{j} .
\end{aligned}
$$

$$
\begin{aligned}
\phi(p) & =\phi_{j}^{*}(p) & & \text { if } p \in U_{j}, j=1, \ldots, k, \\
& =p & & \text { if } p \in R^{n}-\bigcup K_{j} .
\end{aligned}
$$

Then $\phi$ is the required diffeomorphism.

In the rest of this section, unless otherwise specified, $U$ will denote an open subset of $R^{n}(n \geqq 2)$ satisfying condition (*).

LEMMA 7. Let $T=\left(f_{1}, \ldots, f_{n}\right)$ be a one-one measurable transformation of $U$ onto $U$ such that $f_{j} \in C_{r}(U)$ for $j=1, \ldots, n$. Let $E$ be a measurable subset of

$$
\left\{p \mid J_{\mathrm{ap}}(T, p)>0\right\} \quad\left(\left\{p \mid J_{\mathrm{ap}}(T, p)<0\right\}\right) .
$$

Suppose that $m(E)>a>0$. Then there is $C^{r}$-diffeomorphism $\phi_{a}$ of $U$ onto $U$ such that

$$
m\left(\left\{p \mid T(p)=\phi_{a}(p)\right\} \cap E\right)>a .
$$

Proof. (1) Suppose that $E \subset\left\{p \mid J_{\mathrm{ap}}(T, p)>0\right\}$. Since $f_{j} \in C_{r}(U)$ for $j=1, \ldots, n$, there is a transformation $S: U \rightarrow R^{n}$ of class $C^{r}$ such that $m(\{p \mid T(p) \neq S(p)\})$ $<m(E)-a$. Then $m(\{p \mid T(p)=S(p)\} \cap E)>a$. Let $K$ be a compact set contained in $E \cap\{p \mid T(p)=S(p)\}^{*}$ and such that $m(K)>a$. From the first property of $K$ and Proposition $1(1)$, we have $J(S, p)=J_{\mathrm{ap}}(T, p)>0$ for every $p$ in $K$. And, since $S|K=T| K, S \mid K$ is one-one. Thus, since $K$ is compact, $S \mid K$ is a homeomorphism. Let $V=\{p \in U \mid J(S, p)>0\}$. Then, by Lemma 1 , there is an open set $W$ such that $K \subset W \subset V$ and $S \mid W$ is a $C^{r}$-diffeomorphism. And $W$ can be chosen so that $S[W] \subset U$. 
Now let $\mathscr{V}$ be the family of all $B(p, e)$ contained in $W$ for which there is $\phi$ in $D_{r}\left(R^{n}\right)$ such that $S|B(p, e)=\phi| B(p, e)$. By Lemma 3, for each $p$ in $W$ there is a positive number $e_{p}$ such that $\left\{B(p, e) \mid 0<e<e_{p}\right\} \subset \mathscr{V}$. Thus $W$ and $\mathscr{V}$ satisfy the hypothesis of Vitali's theorem (see [7, p. 109]). Hence, since $m(W)<\infty$, there is a finite disjoint family $\left\{B\left(p_{j}, e_{j}\right) \mid j=1, \ldots, k\right\}$ contained in $\mathscr{V}$ such that

$$
m\left(W-\bigcup_{j=1}^{k} B\left(p_{j}, e_{j}\right)\right)<m(K)-a .
$$

Let $B_{j}=B\left(p_{j}, e_{j}\right)$ for $j=1, \ldots, k$. Now there is a finite family $\left\{\phi_{j} \mid j=1, \ldots, k\right\}$ contained in $D_{r}\left(R^{n}\right)$ such that $S\left|B_{j}=\phi_{j}\right| B_{j}$ for $j=1, \ldots, k$. Let $K_{a}=K \cap\left[\bigcup_{j=1}^{k} B_{j}\right]$. Then $m\left(K_{a}\right)>a$. And $\left\{\phi\left[B_{j}\right] \mid j=1, \ldots, k\right\}$ is a disjoint family since $S \mid W$ is one-one.

Next, let $\left\{B\left(q_{j}, f_{j}\right) \mid j=1, \ldots, k\right\}$ be a disjoint family contained in

$$
U-\bigcup_{j=1}^{k}\left[B_{j} \cup \phi_{j}\left[B_{j}\right]\right]
$$

Let $B_{j}^{\prime}=B\left(q_{j}, f_{j}\right)$ for $j=1, \ldots, k$. Now, for $j=1, \ldots, k$, let $\alpha_{j}$ be an affine transformation of $R^{n}$ onto $R^{n}$ such that $\alpha_{j}\left[B_{j}\right]=B_{j}^{\prime}$ and $J\left(\alpha_{j}, p\right)>0$ for all $p$ in $R^{n}$. By Lemma 6, there is $\alpha$ in $D_{\infty}\left(R^{n}\right)$ and a compact set $K_{\alpha}$ contained in $U$ such that $\bigcup_{j=1}^{k} B_{j} \subset$ int $K_{\alpha}, \alpha\left|B_{j}=\alpha_{j}\right| B_{j}$ for $j=1, \ldots, k$, and $\alpha(p)=p$ for $p \in R^{n}-K_{\alpha}$.

Now, by Lemma 6, applied to $\left\{\phi_{j} \circ \alpha_{j}^{-1} \mid j=1, \ldots, k\right\},\left\{B_{j}^{\prime} \mid j=1, \ldots, k\right\}$, and $U$, there is $\phi$ in $D_{r}\left(R^{n}\right)$ and a compact set $K_{\phi}$ contained in $U$ such that $\bigcup_{j=1}^{k} B_{j}^{\prime} \subset$ int $K_{\phi}$, $\phi_{j}\left|B_{j}^{\prime}=\left(\phi_{j} \circ \alpha_{j}^{-1}\right)\right| B_{j}^{\prime}$ for $j=1, \ldots, k$, and $\phi(p)=p$ for $p \in R^{n}-K_{\phi}$.

Now let $\phi_{a}=\phi \circ(\alpha \mid U)$. Then $\phi_{a} \in D_{r}(U)$ and $\phi_{a}\left|K_{a}=T\right| K_{a}$. For let $p \in K_{a}$. Then there is a positive integer $j \leqq k$ such that $p \in B_{j}$. So

$$
\phi_{a}(p)=\phi(\alpha(p))=\phi\left(\alpha_{j}(p)\right)=\left(\phi_{j} \circ \alpha_{j}^{-1}\right)\left(\alpha_{j}(p)\right)=\phi_{j}(p)=S(p)=T(p) .
$$

Hence $m\left(\left\{p \mid T(p)=\phi_{a}(p)\right\} \cap E\right) \geqq m\left(K_{a}\right)>a$.

(2) Suppose that $E \subset\{p \mid J(T, p)<0\}$. Since $U$ satisfies condition (*), there is a $C^{\infty}$-diffeomorphism $\theta$ of $U$ onto $U$ such that $J(\theta, p)<0$ for all $p$ in $U$. Let

$$
T_{1}=T \circ \theta=\left(g_{1}, \ldots, g_{n}\right) .
$$

Then $g_{j} \in C_{r}(U)$ for $j=1, \ldots, n$. For, since $f_{j} \in C_{r}(U)$ for $j=1, \ldots, n$, there is a sequence $\left\{S_{k}\right\}$ of $C^{r}$-transformations of $U$ into $R^{n}$ such that

$$
\lim _{k \rightarrow \infty} m\left(\left\{p \mid T(p)=S_{k}(p)\right\}\right)=m(U) .
$$

Then, since $\theta^{-1}$ has property $(N)$ and $m(U)<\infty, \lim _{k \rightarrow \infty} m\left(\theta^{-1}\left[\left\{p \mid T(p)=S_{k}(p)\right\}\right]\right)$ $=m(U)$. But, for each $k, \theta^{-1}\left[\left\{p \mid T(p)=S_{k}(p)\right\}\right]=\left\{p \mid T_{1}(p)=S_{k}(\theta(p))\right\}$. So, for $j=1, \ldots, n, g_{j} \in C_{r}(U)$.

Next, let

$$
A=\left[\bigcup_{k=1}^{\infty}\left[\left\{p \mid T_{1}(p)=S_{k}(\theta(p))\right\}^{*} \cap \theta^{-1}\left[\left\{p \mid T(p)=S_{k}(p)\right\}^{*}\right]\right] \cap \theta^{-1}[E] .\right.
$$


Then $m(A)=m\left(\theta^{-1}[E]\right)>0$. And $A \subset\left\{p \mid J_{\mathrm{ap}}\left(T_{1}, p\right)>0\right\}$. For let $p \in A$. Then there is $i$ such that $J_{\text {ap }}\left(T_{1}, p\right)=J\left(S_{i} \circ \theta, p\right)$ and $J_{\text {ap }}(T, \theta(p))=J\left(S_{i}, \theta(p)\right)$. So

$$
J_{\mathrm{ap}}\left(T_{1}, p\right)=J\left(S_{i} \circ \theta, p\right)=J\left(S_{i}, \theta(p)\right) J(\theta, p)=J_{\mathrm{ap}}(T, \theta(p)) J(\theta, p)
$$

Since $\theta(p) \in E$, both factors on the right are negative. Hence $J_{\mathrm{ap}}\left(T_{1}, p\right)>0$.

Since $\theta$ has property $(N)$ and $m(U)<\infty$, there is a positive number $\delta<m(A)$ such that $m(\theta[F])<m(E)-a$ for every measurable subset $F$ of $U$ with $m(F)<\delta$. Now, applying part (1) to $T_{1}, A$, and $m(A)-\delta$, there is $\psi$ in $D_{r}(U)$ such that $m\left(\left\{p \mid T_{1}(p)=\psi(p)\right\} \cap A\right)>m(A)-\delta$. Then $m\left(\theta\left[\left\{p \mid T_{1}(p) \neq \psi(p)\right\} \cap A\right]\right)<m(E)-a$. Let $\phi_{a}=\psi \circ \theta^{-1}$. Then $\phi_{a}$ is the required diffeomorphism.

REMARK. The diffeomorphism $\phi_{a}$ in Lemma 7 can be chosen so that there is a compact set $K^{a}$ contained in $\left\{p \mid T(p)=\phi_{a}(p)\right\} \cap E$ such that $\phi_{a} \mid\left(U-K^{a}\right)$ is a real analytic diffeomorphism. This is a straightforward application of $[8$, Lemmas 1 and 6$]$ and of [4, Theorem 1.2].

LEMMA 8. Let $U$ be an open subset of $R^{n}$ and let $T$ be a $C^{1}$-transformation of $U$ into $R^{n}$. If $E$ is a measurable subset of $U$ and $J(T, p)=0$ for almost all $p$ in $E$, then $m(T[E])=0$.

See $[6$, p. 339] for a proof of Lemma 8.

Proof of Theorem 2. Theorem 2 will follow from Lemma 7 if we show that the set $Z=\left\{p \mid J_{\mathrm{ap}}(T, p)=0\right\}$ has measure zero. Note first that $J_{\mathrm{ap}}(T, \cdot)$ is a measurable function since the $D_{i}^{\text {ap }} f_{j}$ are measurable functions (see [7, p. 299]). Hence $Z$ is a measurable set. There is a sequence $\left\{S_{k}\right\}$ of $C^{r}$-transformations of $U$ into $R^{n}$ such that $\lim _{k \rightarrow \infty} m\left(\left\{p \mid T(p) \neq S_{k}(p)\right\}\right)=0$. For each $k$, let $Z_{k}=Z \cap\left\{p \mid T(p)=S_{k}(p)\right\}$. Then $m\left(Z-\bigcup_{k=1}^{\infty} Z_{k}\right)=0$. Let $k$ be a positive integer. Then, by Proposition 1(1), we have $J\left(S_{k}, p\right)=J_{\mathrm{ap}}(T, p)=0$ for all $p$ in $Z_{k}^{*}$. So, by Lemma $8, m\left(S_{k}\left[Z_{k}\right]\right)=0$. Hence $m\left(T\left[\bigcup_{k=1}^{\infty} Z_{k}\right]\right)=0$. Since $T^{-1}$ has property $(N), m\left(\bigcup_{k=1}^{\infty} Z_{k}\right)=0$. Therefore $m(Z)=0$.

Remarks. (1) It can be shown that if Theorem 2 holds for an open subset $U$ of $R^{n}(n \geqq 2)$, then $U$ satisfies condition (*).

(2) If $U$ is a bounded open subset of $R^{n}$ and $\phi$ is a homeomorphism of $U$ onto $U$, then the condition that $\phi^{-1}$ has property $(N)$ means essentially that $\phi^{-1}$ is "absolutely continuous." Explicitly, this means that $\phi^{-1}$ is $e \mathrm{AC}$ in the terminology of [6].

In [9], Whitney proved the following two theorems, the first of which allows us to determine "explicitly" the approximately smooth one-one measurable transformations of $U$ onto $U$.

THEOREM 3 (WhitNey). Let $U$ be an open subset of $R^{n}$. Then $f \in C_{1}(U)$ if and only if, for $j=1, \ldots, n, D_{j}^{\mathrm{ap}} f(p)$ exists for almost all $p$ in $U$. 
TheOREM 4 (WhITNEY). Let $r$ be a positive integer and let $f$ be a real valued function defined on the open subset $U$ of $R^{n}$. Suppose that

(1) $f$ has partial derivatives of order $\leqq r$ everywhere on $U$ and

(2) each partial derivative of $f$ of order $r$ has a total derivative almost everywhere on $U$.

Then $f \in C_{r+1}(U)$.

Combining these two theorems with Theorem 2, we have the two of the most important results of this paper.

THEOREM 5. Let $T=\left(f_{1}, \ldots, f_{n}\right)$ be a one-one measurable transformation of $U$ onto $U$. Then $T$ is approximately smooth if and only if

(1) for $j, k=1, \ldots, n, D_{j}^{\mathrm{ap}} f_{k}(p)$ exists for almost all $p$ in $U$,

(2) either $J_{\mathrm{ap}}(T, p) \geqq 0$ for almost all $p$ in $U$ or $J_{\mathrm{ap}}(T, p) \leqq 0$ for almost all $p$ in $U$, and

(3) $T^{-1}$ has property $(N)$.

Furthermore, both $T$ and $T^{-1}$ are approximately smooth if and only if $T$ satisfies (1), (2), (3), and

(4) $T$ has property $(N)$.

If T satisfies (1), (2), (3), and (4), then there is a sequence $\left\{\phi_{i}\right\}$ of $C^{1}$-diffeomorphisms of $U$ onto $U$ such that

$$
\lim _{i \rightarrow \infty} m\left(\left\{p \mid T(p) \neq \phi_{i}(p) \text { or } T^{-1}(p) \neq \phi_{i}^{-1}(p)\right\}\right)=0 .
$$

THEOREM 6. Let $r$ be a positive integer. If $T=\left(f_{1}, \ldots, f_{n}\right)$ is a one-one measurable transformation of $U$ onto $U$ such that

(1) for $j=1, \ldots, n, f_{j}$ has partial derivatives of order $\leqq r$ everywhere on $U$,

(2) for $j=1, \ldots, n$, each partial derivative of $f_{j}$ of order $r$ has a total differential almost everywhere on $U$,

(3) either $J(T, p) \geqq 0$ for almost all $p$ in $U$ or $J(T, p) \leqq 0$ for almost all $p$ in $U$, and

(4) $T^{-1}$ has property $(N)$,

then $T$ is approximately smooth of order $r+1$.

REMARK. If $n=2$ in Theorem 6, then $T$ is necessarily a homeomorphism and (3) follows from (1) (see [6]).

Another corollary to Theorem 2 is the following.

THEOREM 7. Let $T$ be a one-one measurable transformation of $U$ onto $U$. If $T$ is approximately smooth of order $r$ for every positive integer $r$, then $T$ is approximately smooth of order $\infty$.

This follows from Theorem 1 and Theorem 2 and the following lemma.

Lemma 9. If $U$ is an open subset of $R^{n}$, then $C_{\infty}(U)=\bigcap_{j=1}^{\infty} C_{j}(U)$. 
Proof. Suppose that $f \in \bigcap_{j=1}^{\infty} C_{j}(U)$ and let $\varepsilon>0$. For each positive integer $j$ choose $g_{j}: U \rightarrow R$ of class $C^{j}$ such that $m\left(\left\{p \mid f(p) \neq g_{j}(p)\right\}\right)<\varepsilon / 2^{j}$. Let

$$
A=\left\{p \mid f(p)=g_{j}(p) \text { for } j=1,2, \ldots\right\} .
$$

Then $m(U-A)<\varepsilon$.

Now let $A_{0}=A$. For any integer $j \geqq 0$, having defined $A_{j}$, let $A_{j+1}=A_{j}^{*}$. Then let $A_{\infty}=\bigcap_{j=1}^{\infty} \cdot A_{j}$. Note that $m\left(U-A_{\infty}\right)<\varepsilon$.

Now, using Proposition 1(2), it is easy to show by induction that

$$
D_{\left(k_{1}, \ldots, k_{n}\right)} g_{s}(p)=D_{\left(k_{1}, \ldots, k_{n}\right)} g_{t}(p)
$$

for all $p$ in $A_{j}$ whenever $s$ and $t$ are positive integers $\geqq j$ and $\sum_{i=1}^{n} k_{i} \leqq j$. So (*) holds for all $p$ in $A_{\infty}$ whenever $s$ and $t$ are positive integers such that

$$
\sum_{i=1}^{n} k_{i} \leqq \min (s, t)
$$

Now let $F$ be a closed set contained in $A_{\infty}$ such that $m(U-F)<\varepsilon$. For every positive integer $n$ and every $n$-tuple $\left(k_{1}, \ldots, k_{n}\right)$ of nonnegative integers define $f_{\left(k_{1}, \ldots, k_{n}\right)}$ by $f_{\left(k_{1}, \ldots, k_{n}\right)}(p)=D_{\left(k_{1}, \ldots, k_{n}\right)} g_{j}(p)$ for $p$ in $F$ where $\sum_{i=1}^{n} k_{i} \leqq j$. From the preceding paragraph it follows that the $f_{\left(k_{1}, \ldots, k_{n}\right)}$ are well defined and that $f$ is of class $C^{\infty}$ on $F$. Then, by [8, Theorem 1], there is a function $h: R^{n} \rightarrow R$ of class $C^{\infty}$ such that $h|F=f| F$. Hence $f \in C_{\infty}(U)$.

REMARK. Theorem 2 is false for $R$. For define $f$ by

$$
\begin{aligned}
f(x) & =x+1 / 2 & & \text { if } 0<x<1 / 2, \\
& =x-1 / 2 & & \text { if } 1 / 2<x<1, \\
& =1 / 2 & & \text { if } x=1 / 2 .
\end{aligned}
$$

Then $f$ is a one-one measurable transformation of $I$ onto $I$ such that $f \in C_{\infty}(I)$, $f^{\prime}(x)=1$ for almost all $x$ in $I$, and $f^{-1}$ has property $(N)$. But, if $g$ is a homeomorphism of $I$ onto $I$, then $m(\{x \mid f(x)=g(x)\}) \leqq 1 / 2$. (See [1, p. 272].)

However, the following statement can be proved.

THEOREM 8. Let $\phi$ be a homeomorphism of I onto I. Then $\phi$ is approximately smooth of order $r$ if and only if

(1) $\phi \in C_{r}(I)$ and

(2) $\phi^{-1}$ has property $(N)$.

3. In this section we show that Theorem 2 is the "best possible" theorem. First we need the following definitions.

A modulus of continuity is a monotone nondecreasing function $\sigma$ defined for all nonnegative real numbers such that $\lim _{x \rightarrow 0+} \sigma(x)=\sigma(0)=0$.

Definition 6. Let $A$ be a subset of $R^{n}$ and let $\sigma$ be a modulus of continuity. Let $C(A, \sigma)$ denote the set of all functions $f: A \rightarrow R$ such that $\|f(p)-f(q)\|$ $\leqq \sigma(\|p-q\|)$ for all $p$ and $q$ in $A$.

We denote the set of all real-valued continuous functions defined on $[0,1]$ by $C[0,1]$. 
TheOREM 9 (GofFMAN). For every sequence $\left\{\sigma_{j}\right\}$ of moduli of continuity, there exist $f$ in $C[0,1]$ such that $m(\{x \mid f(x)=g(x)\})=0$ for all $g$ in $\bigcup_{j=1}^{\infty} C\left([0,1], \sigma_{j}\right)$.

See [2, pp. 741-744] for the proof of Theorem 9. Using a slight modification of the proof of $[2$, Corollary 3$]$, we prove the following statement.

Proposition 2. Let $r$ be a positive integer and let $\left\{\sigma_{j}\right\}$ be a sequence of moduli of continuity.

(1) There is a $C^{r}$-diffeomorphism $\phi_{r}$ of $[0,1]$ onto $[0,1]$ such that if $g:[0,1] \rightarrow R$ is a class $C^{r}$ and $g^{(r)} \in \bigcup_{j=1}^{\infty} C\left([0,1], \sigma_{j}\right)$, then

$$
m\left(\bigcup_{k=0}^{r}\left\{x \mid \phi_{r}^{(k)}(x)=g^{(k)}(x)\right\}\right)=0 .
$$

(2) If $\phi_{r}$ is as in (1), if $g: I \rightarrow R$ is of class $C^{r}$ and if $g^{(r)} \in \bigcup_{j=1}^{\infty} C\left(I, \sigma_{j}\right)$, then (**) holds.

Proof. First, suppose that $r=1$. By Theorem 9 , there is $f$ in $C[0,1]$ such that $m(\{x \mid f(x)=w(x)\})=0$ if $w \in \bigcup_{i, j=1}^{\infty} C\left([0,1], i \sigma_{j}\right)$. Choose positive numbers $a_{0}$ and $b_{0}$ so that $f(x)+b_{0}>0$ for all $x$ in $[0,1]$ and so that $\int_{0}^{1} a_{0}\left(f+b_{0}\right) d m=1$. Define $\phi_{1}$ by $\phi_{1}(x)=\int_{0}^{x} a_{0}\left(f+b_{0}\right) d m$ for $x$ in $[0,1]$. Then $\phi_{1}$ is a $C^{1}$-diffeomorphism of $[0,1]$ onto $[0,1]$. Now suppose that $g:[0,1] \rightarrow R$ is of class $C^{1}$ and $g^{\prime} \in \bigcup_{j=1}^{\infty} C\left([0,1], \sigma_{j}\right)$. Then $a_{0}^{-1} g^{\prime}-b_{0} \in \bigcup_{i, j=1}^{\infty} C\left([0,1], i \sigma_{j}\right)$, so

$$
0=m\left(\left\{x \mid f(x)=a_{0}^{-1} g^{\prime}(x)-b_{0}\right\}\right)=m\left(\left\{x \mid \phi_{1}^{\prime}(x)=g^{\prime}(x)\right\}\right) .
$$

Now, by Proposition 1(2), $\left\{x \mid \phi_{1}(x)=g(x)\right\}^{*} \subset\left\{x \mid \phi_{1}^{\prime}(x)=g^{\prime}(x)\right\}$. Hence

$$
m\left(\left\{x \mid \phi_{1}(x)=g(x)\right\}\right)=0 .
$$

Then it is easily seen that $\phi_{1}$ satisfies (2). And, in the induction step, we replace $f$ by $\phi_{n}$.

COROLlaRY 1. Let $r$ be a positive integer.

(1) There is a $C^{r}$-diffeomorphism $\psi_{\mathrm{r}}$ of $[0,1]$ onto $[0,1]$ such that if $g:[0,1] \rightarrow R$ is of class $C^{r}$ and $\left(g^{(r)}\right)_{\mathrm{ap}}^{\prime}(x)$ exists for almost all $x$ in $[0,1]$, then

$$
m\left(\bigcup_{k=0}^{r}\left\{x \mid \psi_{r}^{(k)}(x)=g^{(k)}(x)\right\}\right)=0 .
$$

(2) If $\psi_{r}$ is as in (1) and if $g: I \rightarrow R$ is of class $C^{r}$ and $\left(g^{(r)}\right)_{\mathrm{ap}}^{\prime}(x)$ exists for almost all $x$ in $I$, then $(* * *)$ holds.

Proof. (1) Let $\sigma_{j}(x)=j x$ for $x \geqq 0, j=1,2, \ldots$ Applying Proposition 2 to $\left\{\sigma_{j}\right\}$, we obtain a $C^{r}$-diffeomorphism $\psi_{r}$ of $[0,1]$ onto $[0,1]$ such that if $w:[0,1] \rightarrow R$ is of class $C^{r}$ and $w^{(r)} \in \bigcup_{j=1}^{\infty} C\left([0,1], \sigma_{j}\right)$, then $m\left(\bigcup_{k=0}^{r}\left\{x \mid \psi_{r}^{(k)}(x)=w^{(k)}(x)\right\}\right)=0$. Now suppose that $g:[0,1] \rightarrow R$ is of class $C^{r}$ and $\left(g^{(r)}\right)_{a p}^{\prime}(x)$ exists for almost all $x$ in $[0,1]$. 
Suppose that $m\left(\left\{x \mid \psi_{r}^{(r)}(x)=g^{(r)}(x)\right\}\right)>0$. Then, by Theorem 3, applied to $g^{(r)} \mid I$, there is a function $v:[0,1] \rightarrow R$ of class $C^{1}$ such that

$$
m\left(\left\{x \mid \psi_{r}^{(r)}(x)=g^{(r)}(x)\right\} \cap\left\{x \mid g^{(r)}(x)=v(x)\right\}\right)>0 .
$$

Next, let $u:[0,1] \rightarrow R$ be a function of class $C^{r+1}$ such that $u^{(r)}=v$. Then $u^{(r)} \in \bigcup_{j=1}^{\infty} C\left([0,1], \sigma_{j}\right)$. So

$$
0=m\left(\left\{x \mid \psi_{r}^{(r)}(x)=u^{(r)}(x)\right\}\right)=m\left(\left\{x \mid \psi_{r}^{(r)}(x)=v(x)\right\}\right) .
$$

Therefore $m\left(\left\{x \mid \psi_{r}^{(r)}(x)=g^{(r)}(x)\right\}\right)=0$. The rest of the proof of part 1 and the proof of part 2 are very similar to the corresponding parts of the proof of Proposition 2.

THEOREM 10. (1) Let $\left\{\sigma_{j}\right\}$ be a sequence of moduli of continuity. Let $r$ be a positive integer and let $j$ and $k$ be integers between 1 and $n$. Then there is a $C^{r}$-diffeomorphism $\alpha$ of $I^{n}$ onto $I^{n}$ such that if $S=\left(f_{1}, \ldots, f_{n}\right)$ is a $C^{r}$-transformation of $I^{n}$ into $R^{n}$ with $D_{j}^{r} f_{k}$ in $\bigcup_{i=1}^{\infty} C\left(I^{n}, \sigma_{i}\right)$, then $m(\{p \mid \alpha(p)=S(p)\})=0$. If, in addition, $S$ is a $C^{r_{-}}$ diffeomorphism of $I^{n}$ onto $I^{n}$, then $m\left(\left\{p \mid \alpha(p)=S(p)\right.\right.$ or $\left.\left.\alpha^{-1}(p)=S^{-1}(p)\right\}\right)=0$.

(2) Let $r, j$, and $k$ be as in (1). Then there is a $C^{r}$-diffeomorphism $\beta$ of $I^{n}$ onto $I^{n}$ such that if $S=\left(f_{1}, \ldots, f_{n}\right)$ is a $C^{r}$-transformation of $I^{n}$ into $R^{n}$ and if $D_{j}^{\mathrm{ap}}\left(D_{j}^{r} f_{k}\right)(p)$ exists for almost all $p$ in $I^{n}$, then $m(\{p \mid \beta(p)=S(p)\})=0$. If, in addition, $S$ is a $C^{r_{-}}$ diffeomorphism of $I^{n}$ onto $I^{n}$, then $m\left(\left\{p \mid \beta(p)=S(p)\right.\right.$ or $\left.\left.\beta^{-1}(p)=S^{-1}(p)\right\}\right)=0$.

Proof. In this proof we denote Lebesgue measure on $R$ by $m_{1}$ and Lebesgue measure on $R^{n}$ by $m$.

(1) Let $\phi_{r}$ be a $C^{r}$-diffeomorphism of $I$ onto $I$ satisfying the conclusion of Proposition 2(2), relative to $\left\{\sigma_{j}\right\}$. For $s=1, \ldots, n$, define $\alpha_{s}$ by

$$
\begin{array}{ll}
\alpha_{s}\left(x_{1}, \ldots, x_{n}\right)=x_{s} & \text { if } s \neq j, k, \\
\alpha_{k}\left(x_{1}, \ldots, x_{n}\right)=\phi_{r}\left(x_{j}\right) & \text { for }\left(x_{1}, \ldots, x_{n}\right) \in I^{n}, \\
\alpha_{j}\left(x_{1}, \ldots, x_{n}\right)=x_{k} . &
\end{array}
$$

Let $\alpha=\left(\alpha_{1}, \ldots, \alpha_{n}\right)$. Then $\alpha$ is a $C^{r}$-diffeomorphism of $I^{n}$ onto $I^{n}$.

Now let $S=\left(f_{1}, \ldots, f_{n}\right)$ be a $C^{r}$-transformation of $I^{n}$ into $R^{n}$ with

$$
D_{j}^{r} f_{k} \in \bigcup_{i=1}^{\infty} C\left(I^{n}, \sigma_{i}\right) .
$$

Let $y_{1}, \ldots, y_{j-1}, y_{j+1}, \ldots, y_{n}$ be in $I$. Then the function

$$
\hat{f}=f_{k}\left(y_{1}, \ldots, y_{j-1}, \cdot, y_{j+1}, \ldots, y_{n}\right): I \rightarrow R
$$

is of class $C^{r}$ and $\hat{f}^{(r)} \in \bigcup_{i=1}^{\infty} C\left(I, \sigma_{i}\right)$. Since $m_{1}\left(\left\{t \in I \mid \hat{f}(t)=\phi_{r}(t)\right\}\right)=0$, $m_{1}\left(\left\{t \in I \mid f_{k}\left(y_{1}, \ldots, y_{j-1}, t, y_{j+1}, \ldots, y_{n}\right)=\alpha_{k}\left(y_{1}, \ldots, y_{j-1}, t, y_{j+1}, \ldots, y_{n}\right)\right\}\right)=0$.

Then, by Fubini's theorem, $m\left(\left\{p \in I^{n} \mid \alpha_{k}(p)=f_{k}(p)\right\}\right)=0$. Hence

$$
m\left(\left\{p \in I^{n} \mid \alpha(p)=S(p)\right\}\right)=0 .
$$


The last statement of (1) follows since $\alpha$ has property $(N)$. The proof of (2) is the same as the proof of (1) with Corollary 1 substituted for Proposition 2.

4. Examples. In this section $m_{1}$ will denote Lebesgue measure on $R$ and $m$ will denote Lebesgue measure on $R^{2}$.

A homeomorphism of $I$ onto $I$ is either monotone increasing or monotone decreasing on $I$ and hence has a derivative almost everywhere on $I$. In contrast to this, for $n \geqq 2$, a homeomorphism of $I^{n}$ onto $I^{n}$ need not have even approximate partial derivatives almost everywhere on $I^{n}$.

EXAMPLE 1. There is a homeomorphism $\alpha=(u, v)$ of $I^{2}$ onto $I^{2}$ such that

(1) $\alpha$ and $\alpha^{-1}$ have property $(N)$ and

(2) $m\left(\left\{p \mid D_{1}^{\text {ap }} v(p)\right.\right.$ exists $\left.\}\right)=0$.

It follows that if $\psi$ is a $C^{1}$-diffeomorphism of $I^{2}$ onto $I^{2}$, then

$$
m\left(\left\{p \mid \alpha(p)=\psi(p) \text { or } \alpha^{-1}(p)=\psi^{-1}(p)\right\}\right)=0 .
$$

Construction. By [5, Theorem 1] there is $f$ in $C[0,1]$ such that if

$$
A=\left\{x\left|\lim _{h \rightarrow 0} \operatorname{ap}\right| \frac{f(x+h)-f(x)}{h} \mid=\infty\right\},
$$

then $m_{1}(A)=1$. We may assume that $|f(x)|<1 / 2$ for all $x$ in $[0,1]$. Let $u(x, y)=x$ for $(x, y)$ in $I^{2}$ and define $v$ by

$$
\begin{aligned}
v(x, y) & =y+2 f(x) y & & \text { if } 0<x<1,0<y \leqq 1 / 2 \\
& =y+2 f(x)(1-y) & & \text { if } 0<x<1,1 / 2 \leqq y<1 .
\end{aligned}
$$

Let $\alpha=(u, v)$.

Verification. The transformation $\alpha$ is a homeomorphism of $I^{2}$ onto $I^{2}$ since $v(x, \cdot)$ is a homeomorphism of $I$ onto $I$ for all $x$ in $I$.

(1) Let $Z$ be a subset of $I^{2}$ of measure 0 . We may assume that $Z$ is a Borel set. Then $\alpha[Z]$ is a Borel set. Now, by Fubini's theorem, $m_{1}(\{y \mid(x, y) \in Z\})=0$ for almost all $x$ in $I$. Thus $m_{1}(\{v(x, \cdot)(y) \mid(x, y) \in Z\})=0$ for almost all $x$ in $I$ since $v(x, \cdot)$ is a piecewise linear homeomorphism. So $m_{1}(\{y \mid(x, y) \in \alpha[Z]\})=0$ for almost all $x$ in $I$. Hence $m(\alpha[Z])=0$. A similar argument shows that $\alpha^{-1}$ has property $(N)$.

(2) Let $B=\left\{(x, y) \mid \lim _{h \rightarrow 0}\right.$ ap $\left.|(v(x+h, y)-v(x, y)) / h|=\infty\right\}$. Then $\{y \mid(x, y) \in B\}$ $=A \cap I$ for all $x$ in $I$. Hence $m(B)=1$. Therefore $m\left(\left\{p \mid D_{1}^{\mathrm{ap}} v(p)\right.\right.$ exists $\left.\}\right)=0$.

The following example shows that even if a homeomorphism is of class $C^{\prime}$, it may fail to be approximately smooth (however, it does follow that its inverse is approximately smooth).

EXAMPLE 2. There is a homeomorphism $\beta$ of $I$ onto $I$ such that

(1) $\beta$ is of class $C^{1}$,

(2) $\beta^{-1}$ is approximately smooth of order $\infty$, and

(3) $\beta$ is not approximately smooth. 
Construction. Let $F$ be a closed nowhere dense set of positive measure contained in $I$. Let $f$ be a bounded, continuous, nonnegative function defined on $I$ such that $F=\{x \mid f(x)=0\}$ and such that $f \mid(I-F)$ is of class $C^{\infty}$. Define $\beta$ by letting

$$
\beta(x)=\left[\int_{0}^{1} f d m_{1}\right]^{-1} \int_{0}^{x} f d m_{1}
$$

for $x$ in $I$.

Verification. Clearly $\beta$ is of class $C^{1}$ on $I, \beta$ is of class $C^{\infty}$ on $I-F$, and

$$
F=\left\{x \mid \beta^{\prime}(x)=0\right\} \text {. }
$$

Since $F$ is nowhere dense and $f(x)>0$ for $x \in I-F, \beta$ is a homeomorphism of $I$ onto $I$.

(2) First we note that $\beta^{-1} \mid(I-\beta[F])$ is of class $C^{\infty}$. Next, since $\beta^{\prime}(x)=0$ for all $x$ in $F, m_{1}(\beta[F])=0$. And $\beta^{-1} \in C_{\infty}(I)$. For let $\varepsilon>0$. Let $K_{\varepsilon}$ be a compact set contained in $I-\beta[F]$ of measure $>1-\varepsilon$. Then $\beta^{-1}$ is of class $C^{\infty}$ on $K_{\varepsilon}$. So, by [8, Theorem 1], there is $g_{\varepsilon}: I \rightarrow R$ of class $C^{\infty}$ such that $g_{\varepsilon}\left|K_{\varepsilon}=\beta^{-1}\right| K_{\varepsilon}$. Thus $\beta^{-1} \in C_{\infty}(I)$. So, since $\beta$ has property $(N)$, by Theorem $8, \beta^{-1}$ is approximately smooth of order $\infty$.

(3) Since $m_{1}(F)>0$ and $m_{1}(\beta[F])=0, \beta$ is not approximately smooth.

Remarks. (1) If $\beta^{*}$ is defined by $\beta^{*}(x, y)=(\beta(x), y)$ for $(x, y)$ in $I^{2}$, then $\beta^{*}$ has properties analogous to those of $\beta$. This technique applies to Example 3 also.

(2) If $T$ is a one-one measurable transformation of $I^{n}$ onto $I^{n}(n \geqq 2)$ such that $T$ is approximately smooth of order $r$ and $T^{-1}$ is approximately smooth, then $T^{-1}$ is approximately smooth of order $r$.

EXAMPLE 3. There is a homeomorphism $\gamma$ of $I$ onto $I$ such that

(1) $\gamma, \gamma^{-1} \in C_{1}(I)$ and

(2) if $\psi$ is a diffeomorphism of $I$ onto $I$, then

$$
m_{1}\left(\left\{x \mid \gamma(x)=\psi(x) \text { or } \gamma^{-1}(x)=\psi^{-1}(x)\right\}\right)=0 .
$$

Construction. Let $\gamma$ be a homeomorphism of $I$ onto $I$ such that there is a set $A$ contained in $I$ of measure 1 such that $m_{1}(\gamma[A])=0$ (see [7, p. 101]).

Verification. Clearly $\gamma$ satisfies (1) since any homeomorphism of $I$ onto $I$ satisfies (1). Now let $\psi$ be as in (2). Since $m_{1}(A)=1$ and $m_{1}(\gamma[A])=0$, we have $\gamma^{\prime}(x)=0$ for almost all $x$ in $A$ (see [7, p. 126]). Hence $m\left(\left\{x \mid \gamma^{\prime}(x)=\psi^{\prime}(x)\right\}\right)=0$. As in the proof of Proposition 2, $m(\{x \mid \gamma(x)=\psi(x)\})=0$. Similarly,

$$
m\left(\left\{x \mid \gamma^{-1}(x)=\psi^{-1}(x)\right\}\right)=0 .
$$

In Theorem 2 part of the hypothesis is that either $J_{\mathrm{ap}}(T, p) \geqq 0$ for almost all $p$ in $U$ or $J_{\text {ap }}(T, p) \leqq 0$ for almost all $p$ in $U$. Obviously this condition cannot be omitted from the hypothesis if $T$ is assumed merely measurable. The following example shows that this condition cannot be omitted even if $T$ is assumed to be a homeomorphism. 
EXAMPLE 4. There is a homeomorphism $\theta=(u, v)\left[\theta^{-1}=(\bar{u}, \bar{v})\right]$ of $I^{2}$ onto $I^{2}$ such that

(1) $u, v, \bar{u}, \bar{v} \in C_{\infty}\left(I^{2}\right)$,

(2) $\theta$ and $\theta^{-1}$ have property $(N)$, and

(3) $m\left(\left\{p \mid J_{\text {ap }}(\theta, p)>0\right\}\right)>0$ and $m\left(\left\{p \mid J_{\text {ap }}(\theta, p)<0\right\}\right)>0$.

It follows from (3) that neither $\theta$ nor $\theta^{-1}$ is approximately smooth.

Construction. Let $J=\{(x, y) \mid 0 \leqq x \leqq 1,0 \leqq y \leqq 1 / 2\}$ and define $\eta$ by $\eta(x, y)$ $=(1-x, y)$ for $(x, y)$ in int $J$. Then $\eta$ is a $C^{\infty}$-diffeomorphism of int $J$ onto int $J$ such that $J(\eta, p)=-1$ for all $p$ in int $J$. We now need the following material.

DEFINITION 7. A subset $A$ of $I^{2}$ is called sectionally zero-dimensional if, for every line $L$ parallel to either the $x$-axis or the $y$-axis and every $\varepsilon>0$, there is a line $L^{\prime}$ parallel to $L$ such that $\operatorname{dist}\left(L, L^{\prime}\right)<\varepsilon$ and $L^{\prime} \cap A=\varnothing$.

Let $C$ be a simple closed curve contained in $R^{2}$. We denote the bounded component of $R^{2}-C$ by $I(C)$.

Definition 8. A set $P$ contained in $I^{2}$ is called a $p$-set if bdry $P$ is a simple closed curve which is composed of a finite number of line segments each of which is parallel to either the $x$-axis or the $y$-axis and $P=$ bdry $P \cup I$ (bdry $P$ ).

THEOREM 11. A homeomorphism of the unit circle in the plane onto itself has an extension to a homeomorphism of the closed unit disk onto itself which is a $C^{\infty}$ diffeomorphism of the open unit disk.

REMARK. Actually, it follows from [4, Theorem 1.3] that the extension may be chosen so that it is a real analytic diffeomorphism of the open unit disk.

The following is an easy corollary to Theorem 11.

Corollary 2. For $i=1,2$, let $\left\{P_{1}^{i}, \ldots, P_{n}^{i}\right\}$ be a disjoint family of p-sets contained in the interior of the p-set $P_{0}^{i}$. Let $\phi$ be a homeomorphism of bdry $P_{0}^{1}$ onto bdry $P_{0}^{2}$. Then there is a homeomorphism $\phi^{*}$ of $P_{0}^{1}-\bigcup\left\{\right.$ int $\left.P_{j}^{1} \mid j=1, \ldots, n\right\}$ onto $P_{0}^{2}-$ $\bigcup\left\{\right.$ int $\left.P_{j}^{2} \mid j=1, \ldots, n\right\}$, and, for $i=1,2$, an open set $U_{i}$ contained in

$$
P_{0}^{i}-\bigcup\left\{\text { int } P_{j}^{i} \mid j=1, \ldots, n\right\}
$$

such that

(1) for $i=1,2, m\left(U_{i}\right)=m\left(P_{0}^{i}-\bigcup\left\{\right.\right.$ int $\left.\left.P_{j}^{i} \mid j=1, \ldots, n\right\}\right)$,

(2) $\phi^{*} \mid U_{1}$ is a $C^{\infty}$-diffeomorphism of $U_{1}$ onto $U_{2}$,

(3) $\phi^{*} \mid$ bdry $P_{0}^{1}=\phi$, and

(4) $\phi^{*}\left[\right.$ bdry $\left.P_{j}^{1}\right]=$ bdry $P_{j}^{2}$ for $j=1, \ldots, n$.

The following comes directly from the proof of [1, Theorem 1] and will be stated without proof.

There are sectionally zero-dimensional compact sets $S^{1}$ and $S^{2}$ contained in int $J$ such that $m\left(S^{1}\right)>0$ and $\eta\left[S^{1}\right]=S^{2}$. Next, there is a family of sets

$$
\left\{S_{j_{1} \ldots j_{k}}^{i}, P_{j_{1} \ldots j_{k}}^{i}\right\}
$$


defined in the following manner. First, there is a positive integer $t_{1}$; for every $j_{1} \leqq t_{1}$, there is a positive integer $t_{j_{1}}$; for every $j_{1} \leqq t_{1}, j_{2} \leqq t_{j_{1}}$, there is a positive integer $t_{j_{1} j_{2}}$; and, for every positive integer $k>1$, having defined positive integers $t_{j_{1} \ldots j_{k-1}}$, there is, for every $j_{1} \leqq t_{1}, j_{2} \leqq t_{j_{1}}, \ldots, j_{k} \leqq t_{j_{1} \ldots j_{k-1}}$, a positive integer $t_{j_{1} \ldots j_{k}}$. Now, for every $k \geqq 1, j_{1} \leqq t_{1}, j_{2} \leqq t_{j_{1}}, \ldots, j_{k} \leqq t_{j_{1} \ldots j_{k-1}}$, and $i=1,2$, there are sets $S_{j_{1} \ldots j_{k}}^{i}$ and $P_{j_{1} \ldots j_{k}}^{i}$ with the following properties:

(1') $S_{j_{1} \ldots j_{k}}^{i}$ is a sectionally zero-dimensional compact set such that $S_{j_{1}}^{i} \subset S^{i}$ and, for $k>1, S_{j_{1} \ldots j_{k}}^{i} \subset S_{j_{1} \ldots j_{k-1}}^{i}$.

(2') diam $\left(S_{j_{1} \ldots j_{k}}^{i}\right) \leqq(1 / 2)^{k}$ and $\eta\left[S_{j_{1} \ldots j_{k}}^{1}\right]=S_{j_{1} \ldots j_{k}}^{2}$.

(3') For $i=1,2$,

$$
S^{i}=\bigcup\left\{S_{j_{1} \ldots j_{k}}^{i} \mid j_{1} \leqq t_{1}, \ldots, j_{k} \leqq t_{j_{1} \ldots j_{k-1}}\right\} .
$$

(4') $P_{j_{1} \ldots j_{k}}^{i}$ is a $p$-set such that $\operatorname{diam}\left(P_{j_{1} \ldots j_{k}}^{i}\right) \leqq(1 / 2)^{k-1}$.

(5') For $i=1,2$,

is a disjoint family.

$$
\left\{P_{j_{1} \ldots j_{k}}^{i} \mid j_{1} \leqq t_{1}, \ldots, j_{k} \leqq t_{j_{1} \ldots j_{k-1}}\right\}
$$

(6') For $k>1, S_{j_{1} \ldots j_{k}}^{i} \subset$ int $P_{j_{1} \ldots j_{k}}^{i} \subset P_{j_{1} \ldots j_{k}}^{i} \subset$ int $P_{j_{1} \ldots j_{k-1}}^{i}$ and $S_{j_{1}}^{i} \subset$ int $P_{j_{1}}^{i} \subset P_{j_{1}}^{i}$ $\subset$ int $J$.

Note that properties $\left(3^{\prime}\right),\left(4^{\prime}\right)$, and $\left(6^{\prime}\right)$ imply that

(7') For $i=1,2$,

$$
S^{i}=\bigcap_{k=1}^{\infty}\left[\bigcup\left\{P_{j_{1} \ldots j_{k}}^{i} \mid j_{1} \leqq t_{1}, \ldots, j_{k} \leqq t_{j_{1} \ldots j_{k-1}}\right\}\right] .
$$

Next, a homeomorphism $\eta^{*}$ of int $J$ is constructed in the following manner (this is a slight modification of the technique used in the proof of [1, Theorem 1]).

Step 1. For $i=1,2$, let $J_{i 1}$ denote the set $J-\bigcup\left\{\right.$ int $\left.P_{j_{1}}^{i} \mid j_{1} \leqq t_{1}\right\}$. By Corollary 2 there is a homeomorphism $\sigma_{1}$ of $J_{11}$ onto $J_{21}$, and, for $i=1,2$, an open set $U_{i 1}$ contained in $J_{i 1}$ such that

(1.1) $m\left(U_{i 1}\right)=m\left(J_{i 1}\right)$ for $i=1,2$,

(1.2) $\sigma_{1} \mid U_{11}$ is a $C^{\infty}$-diffeomorphism of $U_{11}$ onto $U_{12}$,

(1.3) $\sigma_{1}(p)=p$ for $p$ in bdry $J$, and

(1.4) $\sigma_{1}\left[\right.$ bdry $\left.P_{j_{1}}^{1}\right]=$ bdry $P_{j_{1}}^{2}$ for $j_{1} \leqq t_{1}$.

Step 2. For $i=1,2$, and $j_{1} \leqq t_{1}$, let $K_{j_{1}}^{i}$ denote the set $P_{j_{1}}^{i}-\bigcup\left\{\right.$ int $\left.P_{j_{1} j_{2}}^{i} \mid j_{2} \leqq t_{j_{1}}\right\}$. For $i=1,2$, let $J_{i 2}$ denote the set $\bigcup\left\{K_{j_{1}}^{i} \mid j_{1} \leqq t_{1}\right\}$. For $j_{1} \leqq t_{1}$, apply Corollary 2 to $K_{j_{1}}^{1}, K_{j_{1}}^{2}$ and $\sigma_{1} \mid$ bdry $P_{j_{1}}^{1}$. Hence there is a diffeomorphism $\sigma_{2}$ of $J_{12}$ onto $J_{22}$ and, for $i=1,2$, an open set $U_{i 2}$ contained in $J_{i 2}$ such that

(2.1) $m\left(U_{i 2}\right)=m\left(J_{i 2}\right)$ for $i=1,2$,

(2.2) $\sigma_{2} \mid U_{12}$ is a $C^{\infty}$-diffeomorphism of $U_{12}$ onto $U_{22}$,

(2.3) $\sigma_{2}(p)=\sigma_{1}(p)$ for $p$ in $\bigcup\left\{\right.$ bdry $\left.P_{j_{1}}^{1} \mid j_{1} \leqq t_{1}\right\}$, and

(2.4) $\sigma_{2}\left[\right.$ bdry $\left.P_{j_{1} j_{2}}^{1}\right]=$ bdry $P_{j_{1} j_{2}}^{2}$ for $j_{1} \leqq t_{1}, j_{2} \leqq t_{j_{1}}$.

Let $k$ be an integer greater than 2 and suppose that we have defined homeomorphisms $\sigma_{1}, \ldots, \sigma_{k-1}$. 
Step k. For $i=1,2$, and $j_{1} \leqq t_{1}, \ldots, j_{k-1} \leqq t_{j_{1} \ldots j_{k-2}}$, let $K_{j_{1} \ldots j_{k-1}}^{i}$ denote the set

$$
P_{j_{1} \ldots j_{k-1}}^{i}-\bigcup\left\{\text { int } P_{j_{1} \ldots j_{k}}^{i} \mid j_{k} \leqq t_{j_{1} \ldots j_{k-1}}\right\} \text {. }
$$

For $i=1,2$, let $J_{i k}$ denote the set

$$
\bigcup\left\{K_{j_{1} \ldots j_{k-1}}^{i} \mid j_{1} \leqq t_{1}, \ldots, j_{k-1} \leqq t_{j_{1} \ldots j_{k-2}}\right\} .
$$

For $j_{1} \leqq t_{1}, \ldots, j_{k-1} \leqq t_{j_{1} \ldots j_{k-2}}$, apply Corollary 2 to $K_{j_{1} \ldots j_{k-1}}^{1}, K_{j_{1} \ldots j_{k-1}}^{2}$, and $\sigma_{k-1} \mid$ bdry $P_{j_{1} \ldots j_{k-1}}^{1}$. Hence there is a homeomorphism $\sigma_{k}$ of $J_{1 k}$ onto $J_{2 k}$ and, for $i=1,2$, an open set $U_{i k}$ contained in $J_{i k}$ such that

(k.1) $m\left(U_{i k}\right)=m\left(J_{i k}\right)$ for $i=1,2$,

(k.2) $\sigma_{k} \mid U_{1 k}$ is a $C^{\infty}$-diffeomorphism of $U_{1 k}$ onto $U_{2 k}$,

(k.3) $\sigma_{k}(p)=\sigma_{k-1}(p)$ for $p$ in

and

$$
\bigcup\left\{\text { bdry } P_{j_{1} \ldots j_{k-1}}^{1} \mid j_{1} \leqq t_{1}, \ldots, j_{k-1} \leqq t_{j_{1} \ldots j_{k-2}}\right\},
$$

$$
\sigma_{k}\left[\text { bdry } P_{j_{1} \ldots j_{k}}^{1}\right]=\text { bdry } P_{j_{1} \ldots j_{k}}^{2} \text { for } j_{1} \leqq t_{1}, \ldots, j_{k} \leqq t_{j_{1} \ldots j_{k-1}} .
$$

By induction we have a sequence $\left\{\sigma_{k}\right\}$ of homeomorphisms. Now define $\eta *$ by

And define $\theta$ by

$$
\begin{aligned}
\eta^{*}(p) & =\eta(p) \quad \text { if } p \in S^{1}, \\
& =\sigma_{k}(p) \quad \text { if } k \geqq 1 \text { and } p \in J_{1 k} .
\end{aligned}
$$

$$
\begin{aligned}
\theta(p) & =p & & \text { if } p \in I^{2}-J \\
& =\eta^{*}(p) & & \text { if } p \in J .
\end{aligned}
$$

Verification. The proof that $\eta^{*}$ is a homeomorphism of $J$ onto $J$ is given in [1].

Now, for $i=1,2$, let $U_{i}=\bigcup\left\{U_{i k} \mid k=1,2, \ldots\right\}$. Then from (k.1), we have $m\left(U_{i}\right)=m\left(J-S^{i}\right)$ for $i=1,2$. And, from (k.2), we have $\eta^{*} \mid U_{1}$ is a $C^{\infty}$-diffeomorphism of $U_{1}$ onto $U_{2}$.

We now show that $\theta$ has properties (1), (2), and (3).

(1) Let $\varepsilon>0$. Let $A_{1}, A_{2}$, and $A_{3}$ be compact sets such that $A_{1} \subset I^{2}-J, A_{2} \subset S_{1}$, $A_{3} \subset U_{1}$, and such that if $A=A_{1} \cup A_{2} \cup A_{3}$, then we have $m\left(I^{2}-A\right)<\varepsilon$. There are disjoint open sets $V_{2}$ and $V_{3}$ such that $A_{2} \subset V_{2} \subset$ int $J$ and $A_{3} \subset V_{3} \subset U_{1}$. Let $V=\left(I^{2}-J\right) \cup V_{2} \cup V_{3}$. If $\phi$ is defined by

$$
\begin{aligned}
\phi(p) & =p & & \text { if } p \in I^{2}-J, \\
& =\eta(p) & & \text { if } p \in V_{2}, \\
& =\eta^{*}(p) & & \text { if } p \in V_{3},
\end{aligned}
$$

it follows that $\phi$ is of class $C^{\infty}$ on $V$. And, since $\theta|A=\phi| A$, we have that $u$ and $v$ are of class $C^{\infty}$ on $A$. Thus, by [8, Theorem 1], there are functions $u^{*}$ and $v^{*}$ of class $C^{\infty}$ on $I^{2}$ such that $u^{*}|A=u| A$ and $v^{*}|A=v| A$. Thus $u$ and $v$ are in $C_{\infty}\left(I^{2}\right)$. A similar argument shows that $\bar{u}$ and $\bar{v}$ are in $C_{\infty}\left(I^{2}\right)$.

(2) Since $\theta\left|S^{1}, \theta\right|\left(I^{2}-J\right), \theta \mid U_{1}$, and $\theta \mid\left[J-\left(S^{1} \cup U_{1}\right)\right]$ all have property $(N)$, it follows that $\theta$ has property $(N)$. Similarly, $\theta^{-1}$ has property $(N)$. 
(3) Since $I^{2}-J \subset\{p \mid J(\theta, p)=1\}$, it follows that $m\left(\left\{p \mid J_{\text {ap }}(\theta, p)>0\right\}\right) \geqq m\left(I^{2}-J\right)$ $>0$. And, since $\theta\left|S^{1}=\eta\right| S^{1}$, by Proposition $1(1)$, we have $J_{\mathrm{ap}}(\theta, p)=J(\eta, p)=-1$ for $p$ in $\left(S^{1}\right)^{*}$. Thus $m\left(\left\{p \mid J_{\mathrm{ap}}(\theta, p)<0\right\}\right) \geqq m\left(\left(S^{1}\right)^{*}\right)>0$.

Remark. Let $\psi=\left(f_{1}, \ldots, f_{n}\right)$ be a homeomorphism of $I^{n}$ onto $I^{n}(n \geqq 2)$ such that, for $1 \leqq j \leqq n, f_{j}$ has partial derivatives almost everywhere on $I^{n}$. If $n=2$, then either $J(\psi, p) \geqq 0$ for almost all $p$ in $I^{n}$ or $J(\psi, p) \leqq 0$ for almost all $p$ in $I^{n}$. If $n \geqq 3$ and, in addition, $\psi$ has a weak total differential almost everywhere on $I^{n}$, then the same statement holds. We do not know whether this is true for $n \geqq 3$ if we do not assume that $\psi$ has a weak total differential almost everywhere on $I^{n}$. See [6] for a proof of each of these statements.

\section{REFERENCES}

1. C. Goffman, One-one measurable transformations, Acta Math. 89 (1953), 261-278.

2. - Approximation of non-parametric surfaces of finite area, J. Math. Mech. 12 (1963), 737-745.

3. W. Huebsch and M. Morse, Schoenflies extensions without interior differential singularities, Ann. of Math. (2) 76 (1962), 18-54.

4. —, Analytic diffeomorphisms approximating $C^{m}$-diffeomorphisms, Rend. Circ. Mat. Palermo (2) 11 (1962), 25-46.

5. V. Jarnik, Sur les nombres derives approximatifs, Fund. Math. 22 (1934), 4-16.

6. T. Rado and P. V. Reichelderfer, Continuous transformations in analysis, Springer, Berlin, 1955.

7. S. Saks, Theory of the integral, 2nd ed., translated by L. C. Young, Warsaw-Lwow, 1937.

8. H. Whitney, Analytic extensions of differentiable functions defined in closed sets, Trans. Amer. Math. Soc. 36 (1934), 63-89.

9. —_ On totally differentiable and smooth functions, Pacific J. Math. 1 (1951), 143-159.

University OF New MeXico, Albuquerque, New MeXico 